\title{
La metaevaluación docente en la Universidad Santo Tomás: el instrumento de evaluación.
}

\section{The teacher meta-evaluation at Santo Tomás University: the evaluation instrument.}

\author{
Carlos Orozco ${ }^{1}$, Lida Rubiela Fonseca Gómez², lliana Fernández Peña ${ }^{3}$. \\ ${ }^{1}$ Docente Universidad Santo Tomás, Colombia, carlos.orozco@usantotomas.edu.co, ${ }^{2}$ Docente \\ Universidad Santo Tomáslidafonseca@usantotomas.edu.co, ${ }^{3}$ Docente Universidad Santo Tomás, \\ ilianafernandez@usantotomas.edu.co
}

\section{RESUMEN}

La metaevaluación docente en la Universidad Santo Tomás (USTA) es un proceso de gestión que se articula a las funciones sustantivas de docencia, investigación y responsabilidad social, en coherencia con el análisis de varias categorías. La evaluación del desempeño docente se realiza por tres actores: estudiante, docente (autoevaluación) y Decano, quienes ofrecen una cualificación en cada semestre académico. La revisión de resultados obtenidos por los docentes permite dar una mirada integral a procesos que acompañan la calidad de la educación a nivel de facultades (carreras), divisiones (conjunto de facultades similares) y finalmente al interior de la Universidad, sin embargo, constituye uno de los procesos más controversiales, que ha repercutido en la falta de aceptación en los docentes, por lo que se realiza una investigación que parte del siguiente problema científico: ¿Qué instrumento de evaluación generar en la USTA que responda a las características contextuales de los docentes en pro de la calidad de la docencia universitaria?, con el objetivo de caracterizar los resultados del instrumento de evaluación docente en los tres actores y la finalidad de, en una segunda etapa, proponer una actualización del modelo de evaluación que se corresponda con las nuevas realidades de estudiantes y profesorado.Los datos analizados corresponden al seguimiento transversal de los últimos tres años en la Institución, cuyos resultados del proceso de metaevaluación institucional muestran que en general las facultades obtienen resultados promedio superiores a 4 . Finalmente, los estudiantes perciben un compromiso del docente y una pertenencia institucional acorde con la filosofía tomasina.

Palabras clave: Metaevaluación; evaluación docente; instrumento de evaluación

\section{ABSTRACT}

Teacher meta-evaluation at Santo Tomás University (USTA) is a managementprocessthatisarticulatedtothe substantive functionsofteaching, research and social responsibility, in coherencewiththeanalysisofseveralcategories. Theevaluationoftheteaching performance iscarriedoutbythreeactors: student, teacher (self-evaluation) and Dean, whooffer a qualification in eachacademicsemester. Thereviewoftheresultsobtainedbytheteachersallowsustotake a comprehensive look at processesthataccompanythequalityofeducation at theleveloffaculties (careers), divisions (set of similar faculties) and finallywithintheUniversity, however, constitutesoneofthemost controversial processes, which has hadanimpactonthelackofacceptanceamongteachers, so thatresearchiscarriedoutbasedonthefollowingscientificproblem: Whatevaluationinstrumenttogenerate in the USTA thatrespondstothe contextual characteristicsofteachers in favor ofthequalityofuniversityteaching ?, withtheobjectiveofcharacterizingtheresultsoftheteachingevaluationinstrument in thethreeactors and thepurposeof, in a secondstage, proposinganupdateoftheevaluationmodelthatcorrespondswiththe new onesrealitiesofstudents and faculty. Theanalyzed data correspondtothe transversal 
monitoringofthelastthreeyears in thelnstitution, whoseresultsoftheinstitutional meta-evaluation show that in general thefacultiesobtainaverageresults superior to 4 . Finally, thestudentsperceive a commitmentoftheteacher and aninstitutionalbelongingwiththe tomasina philosophy.

Keywords: Meta-evaluation; teacher evaluation; evaluation instrument

\section{INTRODUCCIÓN}

La evaluación de los profesores, en la Universidad Santo Tomás, ha venido evolucionando en los últimos tres quinquenios. De manera paulatina se han venido presentando intervenciones significativas, siempre con el ánimo de contribuir en los procesos de mejoramiento de la calidad del servicio educativo que se presta en esta Institución que cuenta con más de 438 años de historia y se constituye como el primer Claustro Universitario de Colombia.

Para el año 2005 la Universidad Santo Tomás inicia un proceso de revisión y ajuste al modelo e instrumento con el cual se ejecutaba la evaluación docente, lo anterior en respuesta a un ejercicio reflexivo y crítico frente a la relevancia que tienen los procesos de evaluación y su incidencia directa, no solo en el desarrollo profesoral y su impacto en el estudiantado, sino también en el impacto de ésta en el cumplimiento de metas institucionales que respondan a los procesos de calidad establecidos.

Fruto de esta revisión y ajuste se creó un grupo multidisciplinar, compuesto por diferentes actores de la comunidad académica, entre ellos directivos, decanos, profesores y estudiantes, que, liderados por la Vicerrectoría Académica General a través de la Unidad de Planeación Académica y Perfeccionamiento Docente, inicia la preparación de nuevos instrumentos que a dicha época deberían ser implementados de manera sistematizada. Igualmente, dicha Unidad, desarrolló un documento que, a manera de política institucional en la materia, daría rumbo y orientación a las estrategias, metodologías y acciones a seguir en el futuro, Resultado de este trabajo se presentó y acogió, el documento final, por parte del Consejo Académico Particular en la sesión del día martes 28 de junio de 2005. Para los subsiguientes periodos académicos se implementó la evaluación docente institucional a través de lo normado en dicho documento, incluso a la fecha.

Para 2012, se crea una alianza estratégica entre la Unidad de Desarrollo Curricular y Formación Docente (conocida anteriormente como Unidad de Planeación Académica y Perfeccionamiento Docente) y la Facultad de Estadística, que formulan bajo el marco de un proyecto de investigación unareflexión y "evaluación" de la evaluación docente. Dicha iniciativa es denominada "Metaevaluación del proceso de evaluación docente institucional USTA 2012-2014" cuyo propósito era "generar nuevas y mejores formas de abordar el proceso de evaluación al interior de la Universidad" (USTA, 2010, pág.35) y poner sobre la mesa la validez, pertinencia y fiabilidad del instrumento de evaluación. Además, otros tópicos que estuvieron bajo análisis, fueron la correlación entre los resultados de desempeño docente y el promedio de desempeño de los estudiantes, así como aquellas fortalezas y oportunidades de mejora, que se identifican para el docente, manifestadas por los estudiantes a través de la evaluación.

En sus inicios el proyecto de investigación fue desarrollado en el marco de dos paradigmas de investigación, el primero fue el empírico-analítico, que contempló el análisis cuantitativo a través de técnicas como el índice de confiabilidad Alfa de Cronbach y el Análisis Factorial, éstas permitieron medir la validez y confiabilidad del instrumento. Asimismo, se llevó a cabo un Análisis de Correlación, que permitió determinar si existe relación entre la nota definitiva de los estudiantes y loa nota de la evaluación del docente. Finalmente, en esta etapa temprana de la investigación, se realizó la construcción de unos "índices sintéticos utilizando análisis de componentes principales", este constructo permitió clasificar facultades y docentes de acuerdo, a la percepción de los estudiantes. 
El segundo paradigma fue el comprensivo-interpretativo, que contempló el análisis cualitativo de los criterios y resultados que arrojó el instrumento aplicado y su estrecha relación con el contexto general de la Institución y la percepción de los estudiantes.

El propósito de este ejercicio autocrítico, a través de las técnicas investigativas, es evidenciar los resultados obtenidos a través de la metaevaluación docente al interior de la USTA con el fin de propiciar espacios que permitan la reflexión con fines de dinamización y fortalecimiento del rol docente.

ParaFocault "la evaluación es problematizada y entendida como una técnica de control a través de la cual se establecen itinerarios, escalafones, trayectorias ascensos y remuneraciones, que generan procesos de diferenciación y competencia" (Irigoyen, 2015. pág.134). Bajo esta premisa y con base en la Filosofía Institucional de la Santo Tomás, se diluye la fundamentación ética y política que debería orientar el ejercicio de evaluación docente y se cercena el camino que permita generar acciones de mejora que a posterioriposibiliten el fortalecimiento de la carrera docente en cualquier institución educativa.

Cabe recordar que los procesos evaluativos en su afán de dar respuesta a criterios de calidad "desconocen la mirada contextual y despojan a la evaluación misma de sus componentes esenciales: a quién sirve, a quién trata de beneficiar" (Santos, 2017. pág.473). En ese sentido, cobra importancia la necesidad de mirar al interior de las instituciones con ojos críticos sin perder de vista el contexto propio, por ello el proyecto de metaevaluación de la USTA no solo se dedica a comprobar la valides y fiabilidad instrumental, sino que reconoce otros aspectos importantes como la dedicación, la carga, la naturaleza de los espacios académicos, entre otros, que podrían incidir en el desempeño docente.

Bajo las premisas que se describen en estos párrafos introductorios es que la Universidad Santo Tomás, desde los últimos quince (15) años, viene en la reflexión autocrítica sobre la evaluación docente institucional, se sabe que la objetividad sería el ideal, pero no siempre es la realidad que se vive al interior del proceso de evaluación, en algunas ocasiones se referencia por parte del docente una percepción de mala valoración por parte de sus estudiantes por causales diversos. En consecuencia, el proceso de evaluación de la evaluación (metaevaluación) se rige bajo criterios de validez y fiabilidad de los instrumentos, que científicamente (con uso de las técnicas estadísticas) den margen al error mínimo en los resultados de la evaluación por parte de los estudiantes a sus profesores.

En este orden de ideas, este trabajo da a conocer los resultados que, a través del método científico, arroja el proceso de metaevaluación en el periodo 20161 a 20172 con los respectivos aportes críticos y analíticos que bajo la mirada de la pedagogía problémica (propia del Modelo Educativo Pedagógico de la USTA), permiten el perfeccionamiento continuo a través de propuestas de mejoras, tanto al instrumento como al proceso mismo de evaluación.

\section{METODOLOGÍA}

Para el desarrollo del análisis se contó con la siguiente selección de procedimientos los cuales abordan diferentes métodos y técnicas de tipo mixto con mayor enfoque cuantitativo, a saber: análisis de fiabilidad, análisis de validez, análisis factorial exploratorio, análisis factorial confirmatorio y el análisis descriptivo univariado y multivariado. La población objeto corresponde a todos los docentes de Pregrado con vinculación de tiempo completo, medio tiempo y cátedra laboral de la Universidad Santo Tomás Sede Principal quienes, a través delos instrumentos, fueron evaluados en los periodos académicos comprendidos entre 20161 a 20172 , esta cantidad se presentará en la sección de resultados. Asimismo, la recolección de la información se hace a través de tres instrumentos diseñados y revisados, según las directrices de la Política Docente en la USTA, la cual es vigente a la fecha. La escala de medición de cada ítem de los instrumentos se manea en escala Likert de 0 a 5 , en donde 0 es no se cumple y 5 se cumple plenamente (Dimensión de la Política Docente USTA, 2010. pág. 41). 


\section{RESULTADOS Y DISCUSIÓN}

\section{Desde dónde entendemos la evaluación}

Para dialogar sobre "metaevaluación" en la USTA, es pertinente entender como es pensada la evaluación desde el interior de la Universidad, para esta investigación se concibe que:

"La evaluación es un eje estratégico y dinamizador de la vida académica y del desarrollo del currículo en orden a garantizar que el proceso pedagógico cumpla condiciones de avance y calidad que ayuden a lograr y mantener adecuados niveles de consistencia, flexibilidad e innovación y cumplir los propósitos intencionados en cada una de las etapas y en todo el proceso y dimensiones de la acción educativa" (USTA, Modelo Educativo Pedagógico, 2010, pág. 57-58).

Bajo esta premisa, la evaluación en la Santo Tomás es pilar fundamental de los procesos de enseñanza y aprendizaje, además es factor transversal al currículo y lo nutre en todas sus etapas. De ahí que no solo evidencia resultados en el ámbito del desempeño del profeso, sino que a su vez da reconocimiento a la eficacia de los métodos pedagógicos, a la gestión académica y al adecuado uso de la tecnología.

Los periodos académicos en la USTA son de 16 semanas que, en el primer semestre del año (Lectivo I) van de febrero a mayo y en el segundo semestre (Lectivo II) van de agosto a noviembre. La evaluación docente a nivel de pregrado se realiza una vez por periodo académico;para el lectivo I se realiza las dos últimas semanas de mayo y para el lectivo II se efectúa las dos últimas semanas de octubre. En dichos periodos de tiempo se aplica el instrumento de evaluación, a través de un aplicativo en línea disponible en la dirección electrónica https://evaluaciondocente.usantotomas.edu.co, cuyas credenciales corresponden alas mismas del Sistema Académico SAC tal como se muestra en la Figura1. Ventana de acceso al Sistema de Evaluación Docente de la Universidad Santo Tomás.

Sistema de evaluación docente de la Universidad Santo Tomás

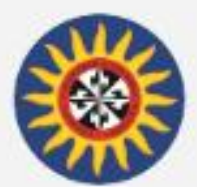

Ingrese para realizar la evaluación docente

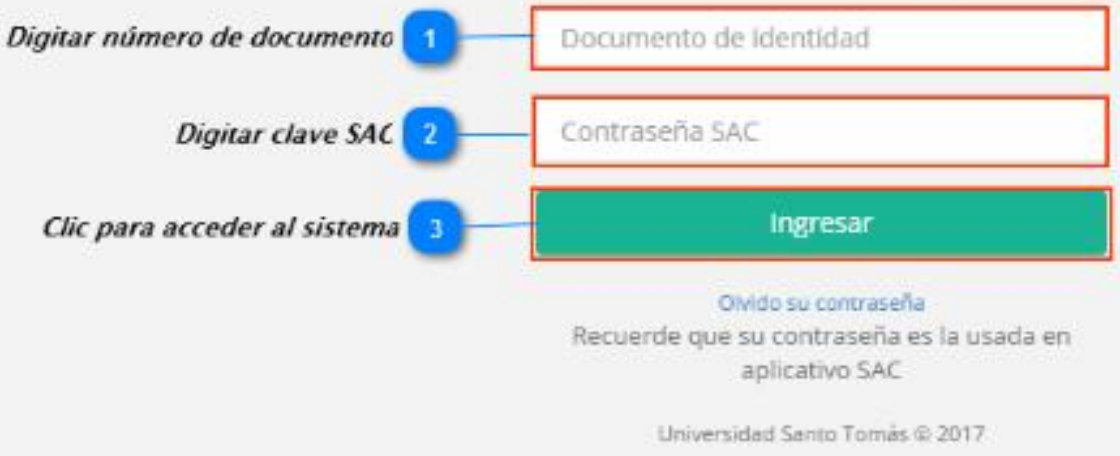

Figura 1. Ventana de acceso al Sistema de Evaluación Docente de la Universidad Santo Tomás. 


\section{Instrumento de evaluación}

Con base en lo expuesto anteriormente en el apartado "Desde donde entendemos la evaluación" de este documento, se presentan: el instrumento de evaluación diseñado por la Universidad Santo Tomás, la escala de calificación de cada uno de los ítems o afirmaciones y el muestreo definido para el análisis.

\section{Tabla 1. Instrumento de evaluación de los estudiantes al profesor}

\begin{tabular}{|c|c|}
\hline Ítem & firmación \\
\hline \multicolumn{2}{|r|}{ Dimensión 1: Programa } \\
\hline 1 & $\begin{array}{l}\text { El docente dio a conocer el programa de la asignatura o espacio académico previamente al } \\
\text { desarrollo de la misma. }\end{array}$ \\
\hline 2 & $\begin{array}{l}\text { El docente hace explicita la importancia de la asignatura o espacio académico en la formación de } \\
\text { los estudiantes. }\end{array}$ \\
\hline 3 & $\begin{array}{l}\text { El desarrollo de la asignatura o espacio académico a la fecha está acorde con la planeación } \\
\text { realizada. }\end{array}$ \\
\hline \multicolumn{2}{|r|}{ Dimensión 2: Disciplina } \\
\hline 5 & $\begin{array}{l}\text { El docente hace uso de la producción académica de su autoría (libros, artículos, prototipos, } \\
\text { módulos, material audiovisual, guías, entre otros) para el desarrollo de la asignatura o espacio } \\
\text { académico. }\end{array}$ \\
\hline 6 & $\begin{array}{l}\text { El docente hace uso de la producción académica institucional para el desarrollo de la asignatura o } \\
\text { espacio académico. }\end{array}$ \\
\hline 7 & El docente demuestra dominio de las temáticas de la asignatura o espacio académico. \\
\hline 8 & $\begin{array}{l}\text { El docente desarrolla en la asignatura o espacio académico, actividades que relacionan diversas } \\
\text { áreas del conocimiento. }\end{array}$ \\
\hline 9 & $\begin{array}{l}\text { El docente estimula el uso de materiales en una segunda lengua para el desarrollo de la } \\
\text { asignatura o espacio académico. }\end{array}$ \\
\hline \multicolumn{2}{|r|}{ Dimensión 3: Pedagógico } \\
\hline 10 & Las explicaciones del docente son comprensibles. \\
\hline 11 & El docente usa ayudas educativas que potencian el aprendizaje. \\
\hline 12 & El docente hace acompañamiento al proceso de aprendizaje. \\
\hline 13 & El docente aplica estrategias metodológicas que favorecen el aprendizaje. \\
\hline 14 & $\begin{array}{l}\text { El docente promueve la consulta de los desarrollos disciplinares recientes en la asignatura o } \\
\text { espacio académico. }\end{array}$ \\
\hline 15 & El docente hace retroalimentación oportuna de las evaluaciones que realiza. \\
\hline 16 & $\begin{array}{l}\text { El proceso de evaluación se realiza con estrategias diversas (pruebas escritas, exposiciones, } \\
\text { tutorías, etc.) }\end{array}$ \\
\hline 17 & Los criterios usados para evaluar son justos y transparentes. \\
\hline \multicolumn{2}{|r|}{ Dimensión 4: Compromiso Institucional } \\
\hline 19 & El docente establece relaciones humanas basadas en el respecto. \\
\hline 20 & El docente implementa estrategias que favorecen la convivencia dentro y fuera del aula. \\
\hline 21 & El docente desarrolla actividades que estimulan la capacidad del trabajo en equipo. \\
\hline 22 & El docente asiste puntualmente a sus clases y a las demás actividades programadas. \\
\hline 24 & El docente promueve las actitudes éticas, creativas y críticas derivadas de la misión institu \\
\hline
\end{tabular}

Fuente: Dimensión de la Política Docente USTA, 2010. pág. 45-46

Es de anotar que la numeración tiene saltos ( 3 al 5, 17 al 19, 23 al 24), esto obedece a que los siguientes ítems no fueron incluidos en la aplicación del instrumento ni en su posterior análisis de resultados, dado que no corresponden a la visión general que tiene esta investigación. Asimismo, los numerales omitidos sólo aplicaban para el Programa de Cultura Física, Deporte y Recreación, que puntualmente son: Criterio 
1 Programa ítem 4 "El manual del deportista fue divulgado oportunamente y es de su total conocimiento, Criterio 2 Pedagógico ítem 18 "Son claros los criterios que usa el docente para definir la participación de los deportistas en los distintos eventos y equipos", Criterio 4 Compromiso Institucionalítem 23 "El docente utiliza el gimnasio de la USTA como soporte de la preparación física general".

En la siguiente tabla se define la escala de calificación bajo la cual los estudiantes evaluaron el grado de cumplimiento de los veintiún ítems o afirmaciones que componen el instrumento de evaluación.

\section{Tabla 2. Criterios de valoración del grado de cumplimiento de cada ítem}

\begin{tabular}{|l|c|}
\hline \multicolumn{1}{|c|}{ Escala } & Calificación \\
\hline Se cumple plenamente & 5 \\
\hline Se cumple en alto grado & 4 \\
\hline Se cumple medianamente & 3 \\
\hline Se cumple en bajo grado & 2 \\
\hline Se cumple insuficientemente & 1 \\
\hline No se cumple & 0 \\
\hline
\end{tabular}

Fuente: Dimensión de la Política Docente USTA, 2010. pág. 41

\section{Muestreo y bases de datos}

Para el análisis del proceso de evaluación se usaron las bases de datos de los periodos académicos comprendidos entre los años 2016 y 2017 (cuatro periodos) cuyos resultados corresponden al total de las evaluaciones recibidas por los docentes durante cada uno de los semestres, a manera de resumen se presenta la tabla 3.

Tabla 3. Total de docentes evaluados por semestre

\begin{tabular}{|c|c|}
\hline Semestre & Cantidad de docentes \\
\hline 20161 & 935 \\
\hline 20162 & 940 \\
\hline 20171 & 972 \\
\hline 20172 & 936 \\
\hline
\end{tabular}

Fuente: Sistema de Evaluación Docente de la Universidad Santo Tomás, 2018

\section{Análisis descriptivo}

Los resultados obtenidos en el proceso de validación del instrumento con el cual se evaluó a los docentes, dejan en evidencia que, en una escala de 0 a 5 (donde 5 es el valor máximo) el promedio es superior a 4 para todos los ítems evaluados, con la excepción del ítem 9 que está orientado a indagar sobre el uso de materiales de enseñanza en segunda lengua por parte de los docentes dentro de sus espacios académicos.

Dicho ítem mostró un alto coeficiente de variación (0.34) y ratifica una necesidad de formación sentida por la mayoría del estamento docente y percibida por gran parte del estudiantado: el dominio de una segunda lengua. El mejoramiento de este criterio pasa por la movilización de estrategias institucionales como los cursos de formación permanente docente en segunda lengua, pero en especial a espacios de cualificación que, a través del uso de estrategias pedagógicas y didácticas busquen el fortalecimiento de las competencias en lengua extranjera que trasciendan más allá de la elaboración de materiales de enseñanza. 
Para la Santo Tomás el dominio de una segunda lengua juega un rol importante en los procesos de acreditación internacional y se constituye en un reto que supera no solo los limites institucionales sino que instala un dialogo alrededor del papel que juega no solo el inglés como opción de segundo idioma, cabe resaltar que según la Revista Portafolio, para 2015 Colombia ocupó el puesto 12 frente a 14 países de América Latina evaluados en competencias en el idioma inglés, algo que ciertamente también se ve reflejado en la evaluación docente.

Al respecto se puede tomar postura critica frente a la importancia de impulsar y visibilizar la oferta de propuestas de segunda lengua diferentes al inglés, como el portugués, francés e incluso lenguajes autóctonos de Colombia, que se ven limitados dado que no se encuentran alineados con la estructura de evaluación de calidad a nivel internacional.

\section{Análisis Factorial Exploratorio}

El Análisis Factorial Exploratorio (AFE) permitió valorar la correlación estadística existente entre las 4 dimensiones del instrumento de evaluación (programa, disciplina, pedagógico y compromiso institucional) y los 21 ítems que integran el mismo (descritos en la tabla 1).

Los resultados cuantitativos indican que, dos de los ítems de la evaluación se asocian a una dimensión diferente a la que pertenecen. Estos son: ítem 7 El docente demuestra dominio de las temáticas de la asignatura o espacio académico e ítem 8 El docente desarrolla en la asignatura o espacio académico actividades que relacionan diferentes áreas del conocimiento. Estosdos ítems pertenecen, en la evaluación docente, a la dimensión disciplinar, pero están siendo asociados por los estudiantes con competencias relacionadas con la dimensión pedagógica.

Bajo este contexto los modelos estadísticos aplicados (AFE, Alfa de Cronbach, AFC) plantean tres modelos de mejora al instrumento de evaluación:

- Modelo 1: El instrumento actual funciona tal como se encuentra diseñado, independientemente de la variabilidad que se observa en los ítems 7 y 8.

- Modelo 2: Se sugiere cambiar la redacción de los ítems 7 y 8, o cambiarlos de dimensión.

- Modelo 3: Se sugiere eliminar los ítems 7 y 8.

Analizando la pertinencia (pros y contras) de cada uno de los modelos y teniendo en cuenta la relevancia de la formulación de ambos ítems cuya percepción está dentro de la dimensión pedagógica pero cuya finalidad es indagar sobre la trasmisión del saber disciplinar se propone reformular el ítem 7 así: El docente se muestra conocedor de su disciplina e invita al aprendizaje de nuevas tendencias teóricas y metodológicas.

En este orden de ideas para el ítem 8 se propone reescribirlo para que sea más comprensible a los estudiantes, siendo la propuesta: El docente desarrolla en la asignatura o espacio académico actividades que permiten el diálogo de la disciplina con otras áreas del conocimiento.

Si bien el instrumento aplicado es adecuado (según el modelo 1), no se puede desconocer la complejidad que implica para el estudiante realizar una valoración por separado entre lo disciplinar y lo pedagógico dado que ambos ámbitos se implementan para transmitir y construir conocimiento. De por si en la práctica áulica estas dos dimensiones son transversales e inherentes a los procesos de enseñanza y aprendizaje, sin embargo, esto deja en evidencia que, no solo en la USTA, sino en la generalidad de Instituciones muchos profesionales asumen funciones docentes sin contar con la suficiente formación en pedagogía o estrategias didácticas, situación que es percibida por los estudiantes a través de la valoración de los ítems 7 y 8. 


\section{Análisis Factorial Confirmatorio}

El Análisis Factorial Confirmatorio (AFC), busca identificar que modelo de medición de validez y de instrumento de evaluación es el mejor. Tal como se indicó en párrafos anteriores, las propuestas fueron:

- Dejar el instrumento que existe, tal como está conformado.

- Modificar el instrumento con base en el Modelo 2 propuesto por el AFE (reescribir ítems 7 y 8 ).

- Eliminar los ítems 7 y 8.

Tabla 4. Índices de ajuste de los diferentes modelos

\begin{tabular}{|l|l|l|l|l|l|l|l|l|l|l|}
\hline & Chisq $^{\mathbf{1}}$ & $\mathbf{D f}^{2}$ & Pvalue $^{\mathbf{3}}$ & Rmsea $^{\mathbf{4}}$ & Ecvi $^{\mathbf{5}}$ & $\mathbf{N f i}^{\mathbf{6}}$ & $\mathbf{C f i}^{\mathbf{7}}$ & Tli $^{\mathbf{8}}$ & Pgfi $^{\mathbf{9}}$ & Pnfi $^{\mathbf{1 0}}$ \\
\hline modelo 1 & 893.563 & 183 & 0 & 0.011 & 0.028 & 0.999 & 0.999 & 0.999 & 0.791 & 0.870 \\
\hline modelo 2 & 766.243 & 183 & 0 & 0.010 & 0.025 & 0.999 & 0.999 & 0.999 & 0.792 & 0.871 \\
\hline modelo 3 & 443.388 & 146 & 0 & 0.008 & 0.015 & 0.999 & 1.000 & 0.999 & 0.768 & 0.853 \\
\hline
\end{tabular}

1Estadístico Chi-Cuadrado.

2Grados de libertad.

$3 \mathrm{P}$ valor de la prueba.

4 Error de aproximación cuadrático. Se sugiere valores aceptables por debajo de 0.08.

5 Índice de validación cruzada esperada. Se sugiere valores cercanos a cero.

6 Índice del ajuste normado. Se sugiere que esté por encima de 0.9.

7 Índice de ajuste comparativo. Valores por encima de 0.9 indican un buen ajuste del modelo.

8índice de Tucker - Lewis. Se sugiere que esté por encima de 0.9 .

gíndice de bondad de ajuste de la parsimonia. Se sugiere valores cercanos a 1.

10 índice de ajuste normado de parsimonia. Valores elevados índica un menor ajuste.

Fuente: Resultados Estadísticos, elaboración propia, 2018

Con relación a este aspecto se puede decirque,a pesar de existir una buena correlación entre los tres modelos, lo que indica que son altamente susceptibles de aplicar sin afectar la tendencia general de los resultados, resulta de vital importancia para el proyecto de investigación, cuyo propósito general es la mejora del mismo proceso de evaluación, atender a las recomendaciones propuestas en el modelo 2.

Lo anterior, en el entendido de que dicho modelo propone unos ajustes, identificados en el Análisis Factorial, para los ítems 7 y 8 que pueden conducir a la mejora del instrumento y por ende del ejercicio de evaluación docente.

\section{Análisis de correlaciónentre la nota definitiva del estudiante y la nota en la evaluación del docente}

Una de las grandes apuestas en términos investigativos es determinar a través de los medios estadísticos si existía correlación entre la nota que obtiene el estudiante en su periodo académico y la nota que este último le otorga al docente en el ejercicio de la evaluación docente.

Para ello, se tuvo en cuenta que las variables del instrumento se respondieran en forma nominal, mientras que la calificación se da de forma continua en la escala de 0 a 5 , con base en ello se realizó el cálculo de las correlaciones de Sperman, las cuales se ajustan para este tipo de contextos y no dependen de supuestos.

Los resultados de esta técnica permiten reconocer que la correlación existente es muy baja (0.17), por lo tanto, se puede indicar que el estudiante evalúa al profesor independientemente de la nota que este último lo otorga en el periodo académico. 
Tabla 5. Correlación entre la nota definitiva del estudiante y cada una de las dimensiones de la evaluación docente

\begin{tabular}{|c|l|l|l|l|}
\hline Dimensión & Programa & Disciplina & Pedagógico & $\begin{array}{l}\text { Compromiso } \\
\text { Institucional }\end{array}$ \\
\hline Correlaciones & 0.12 & 0.10 & 0.17 & 0.17 \\
\hline
\end{tabular}

Fuente: Resultados Estadísticos, elaboración propia, 2018

Teniendo en cuenta el bajo porcentaje de correlación existente $(0.17)$ se identifica una alerta temprana que puede ser atendida en el marco de un nuevo proyecto investigativo queaborde y rastree las percepciones cualitativas (observaciones de los estudiantes) a fin de desarrollar estrategias de acompañamiento docente.

\section{CONCLUSIONES}

1. La metaevaluación ha permitido fomentar escenarios de reflexión sobre el ejercicio áulico, que propuestos desde la política institucional conllevan a un ejercicio interno del cual nacen propuestas de mejora para el proceso de evaluación quea futuro puedenimpactar la dinámica del quehacer docente.

2. El diseño del instrumento, fue construido bajo la concepción tomista de la pedagogía problémica, cuya apuesta va en sentido de la integralidad y la articulación de las dimensiones a evaluar, por ello el ejercicio evaluativo por parte de los estudiantes a sus profesores debe tener en cuenta el dominio disciplinar y la habilidad pedagógica del docente, la identidad institucional y los valores y prácticas esenciales al interior de la Universidad.

3. El reto de desarrollar competencias en segunda lengua, es un objetivo muy importante a plantearse a nivel institucional, este debe ser atendido de manera pronta y prioritaria, no solo para figurar en mediciones internacionales, sino para dar herramientas reales de comunicación a nivel internacional de nuestros profesores y su consecuente aterrizaje en la internacionalización del currículo enseñado.

4. Los procesos de enseñanza y aprendizaje deben caracterizarse por la transversalidad del saber disciplinar y de la habilidad didáctica al interior de un aula de clase, estas dos dimensiones son complejas al momento de implementar instrumentos de evaluación. Por ello, se requiere construir criterios de evaluación que,a pesar de ser leídos e interpretados separadamente, no pierdan su característica de integralidad, en consecuencia, debe existir en el instrumento una correlación entre la competencia profesional y la competencia pedagógica.

5. La baja correlación entre los resultados de la evaluación docente y la evaluación del estudiante, permite identificar que este último (el estudiante) mantiene un criterio de objetividad y legitima si se quiere, los aspectos a partir de los cuales el docente lleva a cabo la evaluación del aprendizaje. No obstante, como se mencionó en líneas anteriores, la baja correlación (0.17) constituye una alerta Institucional, que invita al seguimiento y acompañamiento de los casos particulares, pues en este tipo de aspectos se ponen en cuestión valores fundamentales como la justicia, la equidad y la transparencia.

6. Finalmente, como lo menciona Luis Sime (1998) la evaluación es una autoreflexión crítica que como actividad problematizadora nos lleva a concebir nuevas formas de entender y practicar la evaluación docente, lo que presupone una oportunidad para la mejora.

\section{CONFLICTO DE INTERESES}

Los autores del presente artículo, declaran que no existe potencial conflicto de intereses relacionados con el desarrollo del mismo, ni con los productos o resultados que del ejercicio de investigación se derivan. 
No obstante, si es válido mencionar que los investigadores forman parte del cuerpo docente de la USTA y que, esta última a través de la Vicerrectoría Académica General apoya y formaliza el acto de la metaevaluación a través de un Proyecto de Investigación FODEIN (Fondo de Investigación).

\section{REFERENCIAS BIBLIOGRÁFICAS}

Amigot, P. \& Martínez, L (2015), Procesos de subjetivación en el contexto neoliberal. El caso de la evaluación del profesorado y la investigación universitaria. RASE. Revista de Asociación de Sociología de la Educación, Volumen 8 №2, mayo 2015.

Díaz, F. (2005), Enseñanza situada: Vínculo entre la escuela y la vida. Capítulo 5: La evaluación auténtica centrada en el desempeño: Una alternativa para evaluar el aprendizaje y la enseñanza. México: McGraw Hill.

Revista Portafolio. (2015), Colombia se rajó en dominio del inglés. Noviembre 10 de 2015.

Santos, A. (2017), La gallina no es un águila defectuosa. Organización, liderazgo y evaluación en las instituciones educativas. Serie. Aseguramiento de la Calidad Educativa. Corporación Universitaria Minuto de Dios, Bogotá, 2017.

Sime, L, (1998). Metaevaluación: Ir más allá de la evaluación para volver sobre ella. Educación. Vol VII. № 14. Pontificia Universidad Católica del Perú. Departamento de Educación.

USTA, 2015. Lineamientos para el diseño y la actualización curricular. Ediciones USTA, Bogotá.

USTA, 2010. Modelo Educativo Pedagógico. Bogotá. Ediciones USTA, Colombia.

USTA, 2010. Dimensión de la Política Docente. Bogotá, Colombia. Ediciones USTA, Colombia.

USTA, 2015. Documento Marco Desarrollo Docente. Bogotá. Ediciones USTA, Colombia.

\section{SOBRE LOS AUTORES}

M.Sc. Carlos Alfonso Orozco,Universidad Santo Tomás, Docente Tiempo Completo de la Unidad de Desarrollo Curricular y Formación Docente. M.Sc. Lida Rubiela Fonseca Gómez, Universidad Santo Tomás, Docente Tiempo Completo de la Facultad de Estadística. M.Sc. Iliana Fernández Peña, Universidad Santo Tomás, Docente Tiempo Completo de la Unidad de Desarrollo Curricular y Formación Docente. 